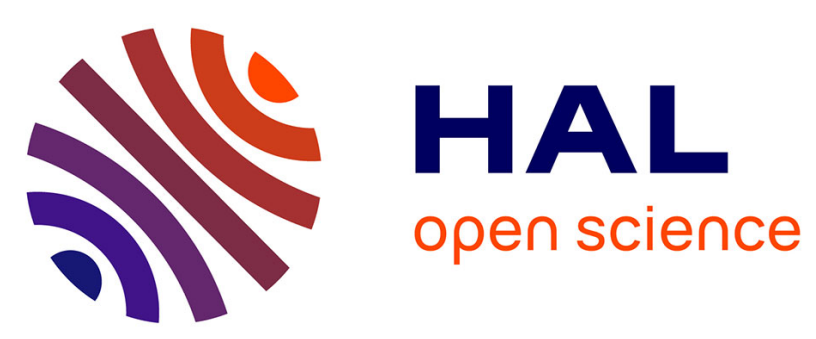

\title{
[MS $]^{2} \mathrm{O}:$ A Multi-scale and Multi-step Ontology for Transformation Processes: Application to Micro-Organisms
}

Juliette Dibie-Barthelemy, Stéphane Dervaux, Estelle Doriot, Liliana L. Ibanescu, Caroline Pénicaud

\section{To cite this version:}

Juliette Dibie-Barthelemy, Stéphane Dervaux, Estelle Doriot, Liliana L. Ibanescu, Caroline Pénicaud. $[\mathrm{MS}]^{2} \mathrm{O}$ : A Multi-scale and Multi-step Ontology for Transformation Processes: Application to MicroOrganisms. 22nd International Conference on Conceptual Structures, Jul 2016, Annecy, France. pp.163-176, 10.1007/978-3-319-40985-6_13 . hal-01357749

HAL Id: hal-01357749

https://hal-agroparistech.archives-ouvertes.fr/hal-01357749

Submitted on 15 Jun 2018

HAL is a multi-disciplinary open access archive for the deposit and dissemination of scientific research documents, whether they are published or not. The documents may come from teaching and research institutions in France or abroad, or from public or private research centers.
L'archive ouverte pluridisciplinaire HAL, est destinée au dépôt et à la diffusion de documents scientifiques de niveau recherche, publiés ou non, émanant des établissements d'enseignement et de recherche français ou étrangers, des laboratoires publics ou privés. 


\title{
$[M S]^{2} O$ - A Multi-Scale and Multi-Step Ontology for Transformation Processes: Application to Micro-Organisms
}

\author{
Juliette Dibie $^{1}$, Stéphane Dervaux ${ }^{1}$, Estelle Doriot ${ }^{1}$, Liliana Ibanescu ${ }^{1}$, and \\ Caroline Pénicaud ${ }^{2}$ \\ 1 UMR MIA-Paris, AgroParisTech, INRA, Université Paris-Saclay, 75005, Paris, \\ France, \\ firstname. lastname@agroparistech.fr \\ 2 UMR GMPA, AgroParisTech, INRA, Université Paris-Saclay, 78850, \\ Thiverval-Grignon, France
}

\begin{abstract}
This paper focuses on the knowledge representation for an interdisciplinary project concerning transformation processes in food science. The use case concerns the production of stabilized micro-organisms performed at INRA (French National Institute for Agricultural Research). Experimental observations are available for some inputs of the production processes, at different steps and at a certain scale. Available data sets are described using different vocabularies and are stored in different formats. Therefore there is a need to define an ontology, called $[M S]^{2} O$, as a common and standardized vocabulary. Users' requirements were defined through competency questions and the ontology was validated against these competency questions. $[M S]^{2} O$ ontology aims to play a key role as the representation layer of the querying and simulation systems of the project. This leads to the possibility of comparing different production scenarios and suggesting improvements.
\end{abstract}

Keywords: domain ontology building, multi-step and multi-scale ontology, transformation processes

\section{Introduction}

There is a challenging need for food companies to design and to control the transformation of raw materials into final products, ensuring their quality while applying appropriate technologies for minimal economic, environmental and social costs. For that, they have to better understand the food production system in order to adopt an eco design approach by considering concomitantly product quality, production process parameters, and its environmental impacts. Such an eco design approach supposes to take advantage of all available data and experts' knowledge for performing the analysis of the production system. However, data have been collected for different purposes, in different sub-domains, at different scales. Data have also been encoded in various formats using heterogeneous 
vocabularies and are processed in different information systems. Moreover, expert's knowledge is often implicit and difficult to acquire. There is therefore a need to uniformly model and store available data and experts' knowledge in order to compare different production scenarios and to perform a cause and effect analysis.

Ontologies are nowadays used as a common and standardized vocabulary for representing concepts and relations from a particular field (e.g. life-science, geography). An ontology is designed to represent the knowledge from a domain in terms of concepts, relations between these concepts and instances of these concepts [8]. Building networks of interconnected ontologies [5] and publishing ontologies on the Linked Open Data (LOD) cloud $^{3}$ should facilitate data integration and data sharing, such as giving access to data from specific disciplines or data produced within specific geographic regions [2]. However, domain ontologies are built for a specific task and it is not easy to reuse them for a different one. Data available at different scales and the challenge to take into account the environmental impact of the food production process cause the knowledge management in food production more complex.

This paper focuses on building an ontology, called $[M S]^{2} O$, for transformation processes and more particularly the production of stabilized micro-organisms, performed at INRA (French National Institute for Agricultural Research) in an inter disciplinary project called LIONES.

A process may be represented as an industrial process [7], a bussiness process [12] or a food transformation process [10]. However, none of these representations was suited to represent a food transformation process described by a set of experimental observations available at different scales. Different Ontology Design Patterns (ODP $)^{4}$ exist as for example the ODP for material transformation [13] or the ODP for life cycle assessment data [9]. As disscused in [3], ODPs bring a promise of compositional modeling and true ontology reuse, but many barriers to their adoption still remain.

Building the ontology from scratch, one of the scenarios of the NeON methodology [5] was used for the construction of $[M S]^{2} O$. Users' requirements were defined through competency questions and the ontology was validated against these competency questions.

$[M S]^{2} O$ core component is implemented in $\mathrm{OWL}^{5}$ and all the available data were structured in files using the $[M S]^{2} O$ vocabulary. The domain component of $[M S]^{2} O$ is under development.

The paper is organized according to the NeOn methodology. In Section 2 the ontology specification is presented, then the ontology conceptualization is detailed in Section 3. In Section 4, we present the ontology implementation and user evaluation. Finally, we conclude in Section 5 and present our further work.

\footnotetext{
${ }^{3}$ http://linkeddata.org

${ }^{4}$ http://ontologydesignpatterns.org

${ }^{5}$ https://www.w3.org/2001/sw/wiki/OWL
} 


\section{Ontology Specification}

This section briefly presents the LIONES Project, then the competency questions expressing users' requirements and finally our ontology specification.

\subsection{LIONES Project}

LIONES Project involves domain experts and computer scientists researchers from INRA, the French National Institute for Agricultural Research. It addresses the issue of modeling semantic LInks between ONtological multi-scalES objects involved in transformation process. LIONES Project is applied to the production of stabilized micro-organisms. Micro-organisms are biological agents which present a large scale of applications in food (e.g. yoghurts, cheese, wine, beer) and non-food (e.g. probiotics, microbial production of chemical molecules, biofuels) domains. The need for concentrated micro-organisms (called starters) to be stabilized and in ready-to-use form is increasing. Their production process [1] is composed of a set of steps or unit operations (e.g. fermentation, cooling, concentration, formulation) that transforms inputs into outputs. The inputs of the system are the raw materials (e.g. micro-organisms, sugar), energy and water. The outputs of the system are the final product, some co-products, energy and effluents. Figure 1 presents the production system for stabilized micro-organism from the domain experts point of view. Two distinct axes were identified: the multi-step axis composed of the different steps of the production process and the multi-scale axis which represents the different scales of the studied product. Moreover, the multi-criteria analysis of the production system should help the experts to guarantee the product quality during the transformation process while reducing economical costs and environmental impact.

The modeling of semantic links in LIONES Project first requires to qualify and represent the multi-scales objects involved in the transformation process. This paper focus on the knowledge representation task of the LIONES Project: the building of $[M S]^{2} O$, a Multi-Scale and Multi-Step Ontology for modeling the production of stabilized micro-organisms.

The available data sets in LIONES Project concerns different steps of the production process at different scales, from the microbial cell components to the target functionality at the population level. Data sets are heterogeneous and sparse and come from many different sources such as databases, EXCEL files, text files, sensor outputs, scientific publications $[11,6,14]$, Master reports, laboratory technical reports. They are gathered for many different purposes by different experts with their own experimental itineraries, vocabularies and technical materiel and methods. Since the experts work on the same domain, reaching a consensus about a common vocabulary was quite easy. Nevertheless (i) extracting and expressing implicit experts' knowledge, (ii) understanding and structuring the whole transformation process with all the involved entities, their properties and interactions using the available data and documents and the implicit experts' knowledge and (iii) identifying the users requirements for data retrieval 


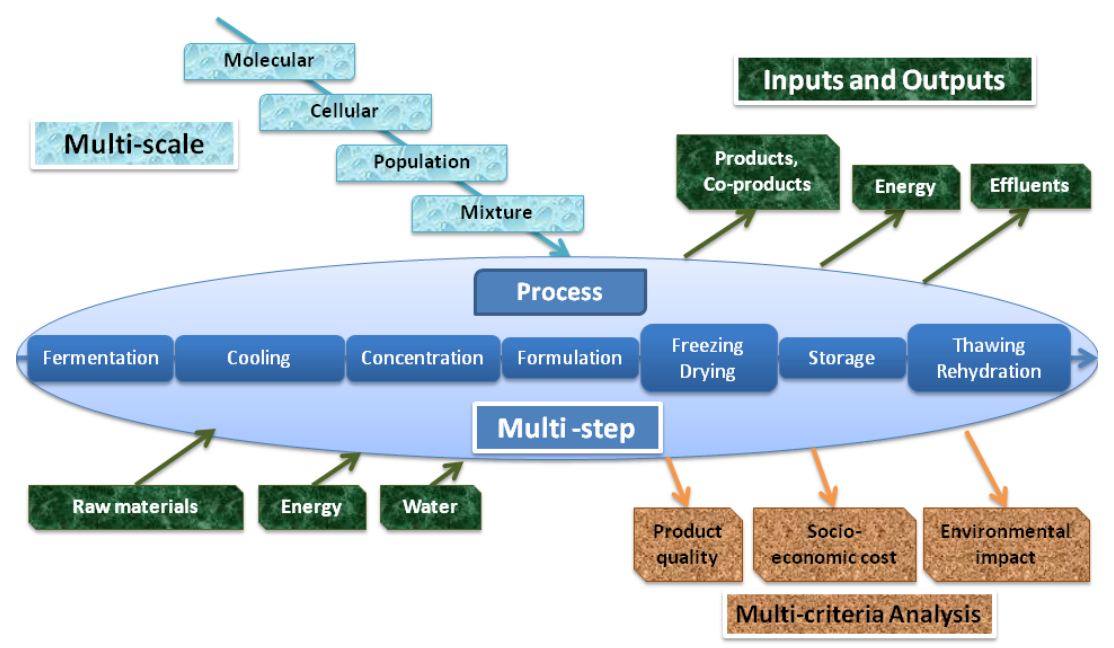

Fig. 1. The production system for stabilized micro-organism

and reasoning were less easier and required extensive conversations with domain experts to model the ontology.

In the next sub-section, we present the users' requirements through competency questions (CQ), which are natural language sentences that express a pattern for a type of questions domain experts expect the ontology to answer.

\subsection{Competency questions}

Domain experts involved in LIONES Project have the following criteria for the product process analysis: the product quality, the environmental impact, and the economical cost. We helped the domain experts to express their users' requirements through a set of competency questions, as for example:

- Competency questions about product quality analysis

$C Q_{1}$ Find all the production conditions of stabilized Lactobacillus delbrueckii subspecies bulgaricus CFL1 that allowed obtaining a specific acidifying activity lower than $20 \mathrm{~min} / \log (\mathrm{CFU} / \mathrm{mL})$.

$C Q_{2}$ Find all the datasets about cultivability losses and membrane integrity of Yarrowia lipolytica during drying.

$C Q_{3}$ Find the Yeast having the most cultivability losses during drying.

$C Q_{4}$ Find which process step was the most damaging for the micro-organisms viability.

$C Q_{5}$ Find all the datasets about drying resistance of Yarrowia lipolytica during the fermentation step.

- Competency questions about environmental impact analysis

$C Q_{6}$ Find all the process steps that are involved in global warming.

$C Q_{7}$ Find the energy consumptions of freeze-drying measured in France during the year 2014. 
- Competency questions about economical cost analysis

$C Q_{8}$ Find all the freeze-drying conditions that allowed producing more than $10 \mathrm{t} /$ month of stabilized bacteria.

The next sub-section presents the ontology specification aiming to answer to the domain experts' competency questions.

\subsection{Ontology Specification}

Using the Figure 1, the available data sets and the related documents, we established, in close collaboration with domain experts, a new representation of the production system as given in Figure 2.

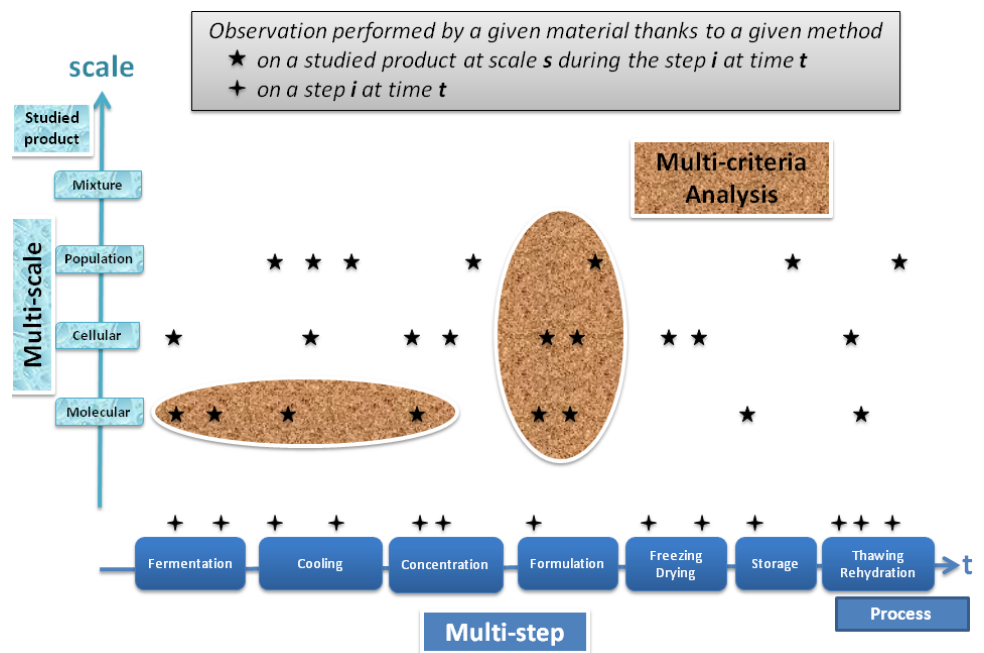

Fig. 2. Global schema of the production system

In this new schema, the two axes of Figure 1 remain: the multi-step axis where the process is described by its different steps and the multi-scale axis where the studied products can be considered at different scales (i.e. as a whole inside a mixture, at the population scale, the cellular or molecular ones). Moreover, we give the following definitions:

D1 a transformation process is composed of steps, non necessarily in sequence (e.g. two steps can occur simultaneously), which transforms inputs into several different outputs;

D2 the representation of a transformation process relies on experimental observations;

D3 experimental observations are performed with a given material according to a given method either on a mixture (i.e. a set of 
combined products) at a certain scale during a certain step (e.g. observations about the membrane integrity of Yarrowia lipolytica performed at its cellular scale during the drying step in $C Q_{2}$ ) or on a step (e.g. observations about energy consumption by the freeze-drying step in $C Q_{7}$ ).

D4 the inputs and outputs of the system (e.g. raw materials, water, coproducts and effluents) of Figure 1 are components of the mixture and can be evaluated through observations on it at different steps and different scales.

D5 The product quality, the economic cost and the environmental impact of Figure 1 can be deduced from attributes associated with the studied products, the steps of the process and the materials.

These five definitions correspond to our understanding of the studied domain and guide our $[M S]^{2} O$ conceptualization.

\section{Ontology Conceptualization}

In this section, we present our Multi Scale and Multi Step Ontology for transformation Processes, $[M S]^{2} O$. Our ontology is designed from scratch by an iterative process in a modular way in order to be able to import and reuse existing ontologies, which will be the next step of our work, as recommended by the $\mathrm{NeOn}$ methodology. $[M S]^{2} O$ is composed of two components: a core component to represent the transformation processes as defined in D1 to D5 and a domain component to represent the production of stabilized micro-organisms. The core component of $[M S]^{2} O$ is composed of the five following modules (see Figure 3):

- a Process module

- a Studied object module

- an Attribute module

- an Observation module

- a Material and Method module

In the next sub sections, we detail its main entities presented in Figure 4, with examples on the production of stabilized micro-organisms. The Material and Method module is not presented in this paper since a complementary expertise is necessary to develop it. Even if its existence seems obvious for the domain experts, this module was not in the initial user requirements. The definition of the competency questions for this module is in progress.

\subsection{The Process module}

According to definition D1, a transformation process is composed of steps, non necessarily in sequence (e.g. two steps can occur simultaneously) which transforms inputs into several different outputs. A process, which corresponds to one experimentation for the domain experts, is composed of several itineraries (see hasForItinerary property in Figure 4). An itinerary is composed of a set of 


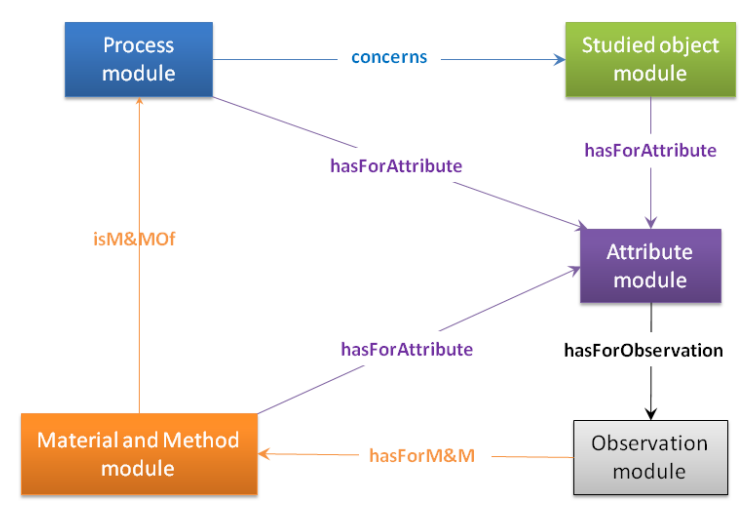

Fig. 3. The five modules of the $[M S]^{2} O$ core component

steps (see hasForStep property in Figure 4) characterized by some experimental conditions linked by time relations (see TimeRelation property on step concept in Figure 4).

Example 1. Let us consider a process on the production and stabilization of the yeast Yarrowia lipolytica performed with two distinct itineraries. The first itinerary is composed of the steps: fermentation, concentration, drying into stove during 75 minutes and storage. The second one is composed of: fermentation, concentration, drying into fluidized bed during 90 minutes and storage.

A step may be composed of substeps (see hasForSubstep property in Figure 4), which are considered as steps. A step is characterized by its material and method (see hasForM\&M property in Figure 4). Figure 5 gives an example of the possible substeps of the fermentation step in different cases.

Each itinerary and step is characterized by the time when it starts (see hasForTimeProperty property in Figure 4).

A step can be compared with the perdurant object Phenomenon in DOLCE ${ }^{6}$ and with the concept Process in $\mathrm{SUMO}^{7}$.

\subsection{The Studied object module}

A studied object may be a product or a mixture. The studied product is the one on which is applied the transformation process (see hasForStudiedObject property between process and product in Figure 4).

Example 2. The studied product of the process presented in Example 1 is the yeast: Yarrowia lipolytica.

\footnotetext{
${ }^{6}$ http://www.loa.istc.cnr.it/old/DOLCE.html

7 http://www.adampease.org/OP/
} 


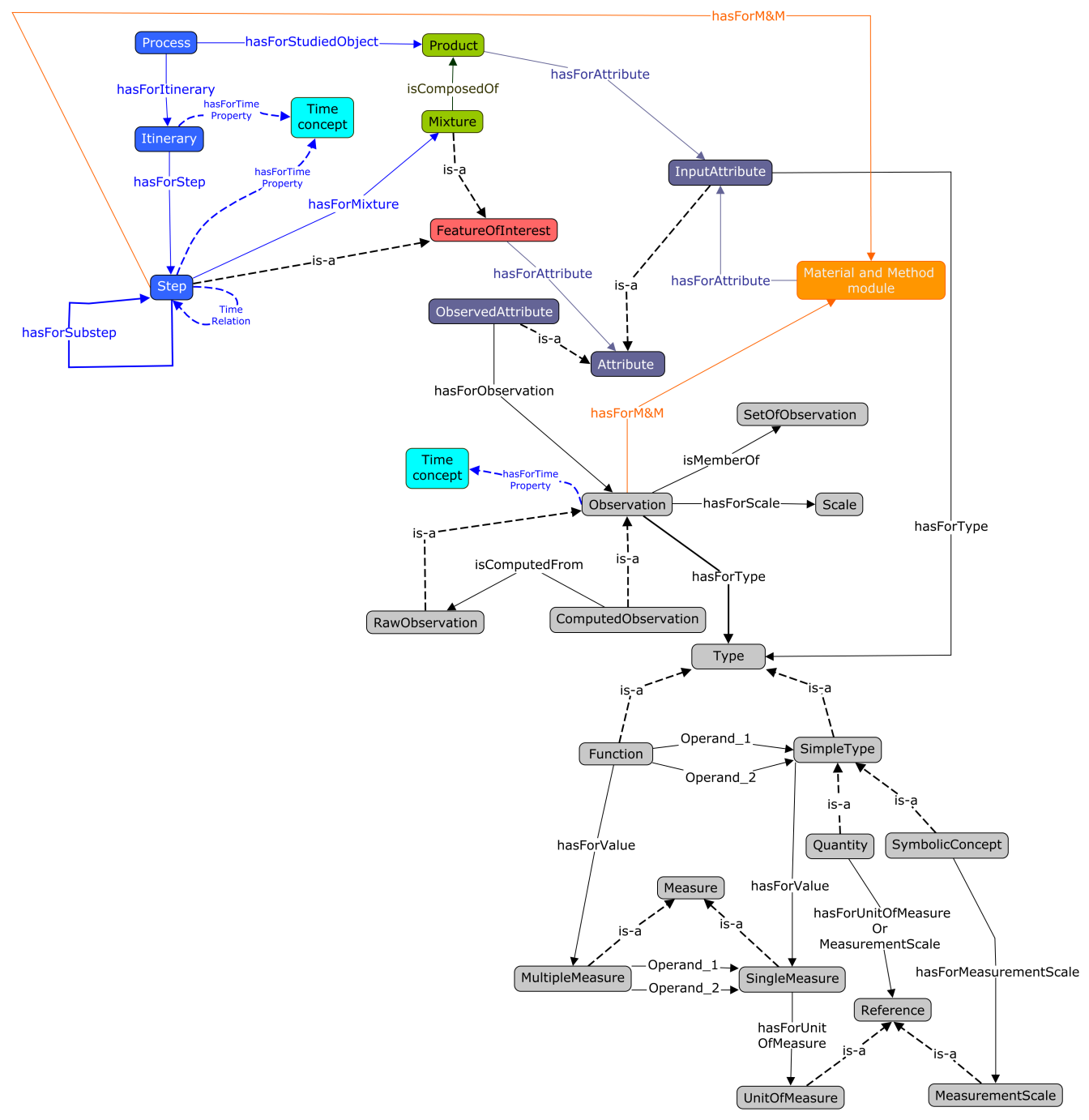

Fig. 4. Detail of the modules Process, Studied object, Attribute and Observation of the $[M S]^{2} O$ core component 


\section{Fermentation}

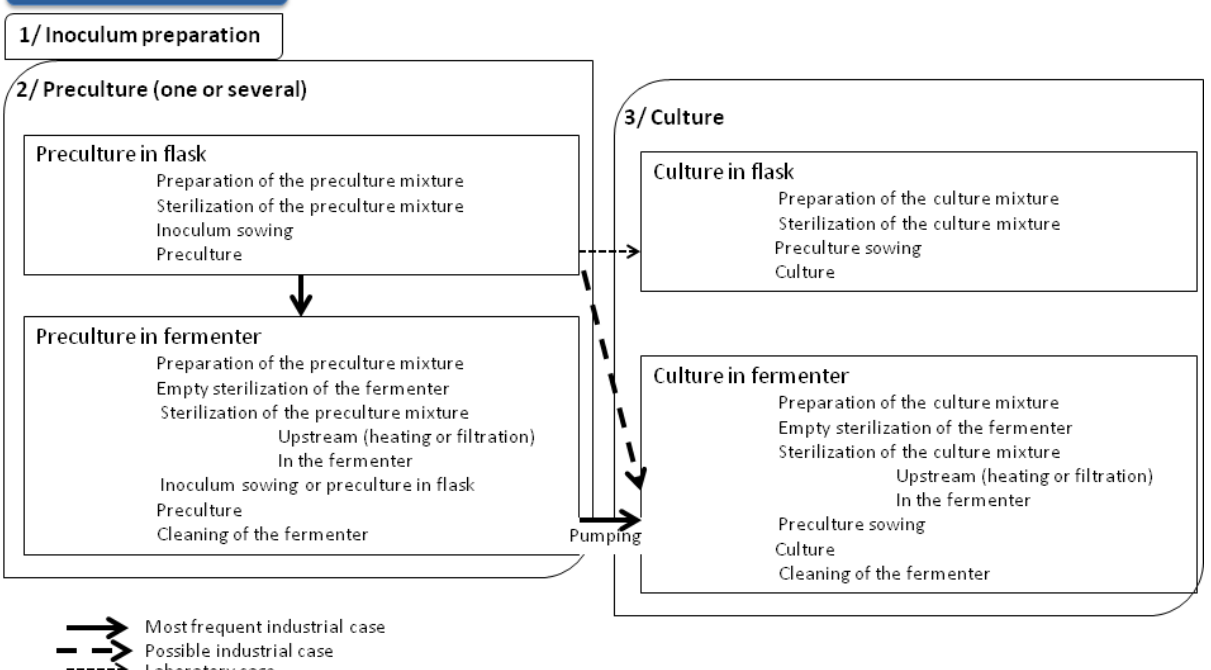

Fig. 5. The possible substeps of the fermentation step

A mixture is a composition of several products (see isComposed0f property in Figure 4), but may be composed of only one product. A mixture is characterized by its composition (e.g. raw materials, water). It contains in particular the studied product. The different steps and sub-steps of an itinerary are applied on mixtures (see hasForMixture property in Figure 4). Let us notice that a step may be applied on several mixtures.

A mixture can be compared with the DOLCE concept Non-Agentive-PhysicalObject.

\subsection{The Attribute module}

The features of interest of our domain are the steps and the studied mixtures. They are characterized by attributes which may have input values or may be observed (see hasForAttribute property between FeatureOfInterest and Attribute in Figure 4). An attribute can therefore be either an input attribute, it is then associated with input values (see Section 3.4), or an observed attribute, it is then associated with observations (see hasForObservation property between ObservedAttribute and Observation in Figure 4). Let us notice that the material and method are also characterized by input attributes (see hasForAttribute property between Material and Method module and Attribute in Figure 4).

Example 3. In order to answer to $C Q_{5}$, we have to consider all the observations associated with the Drying_resistance attribute which characterizes mixtures composed of the yeast Yarrowia lipolytica, during the fermentation step. Let us 

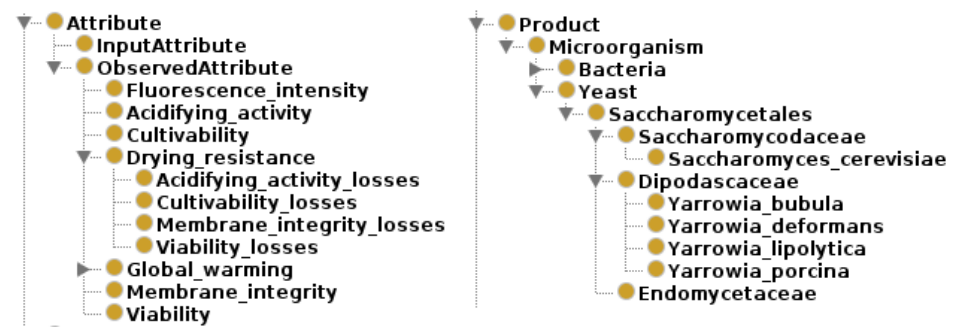

Fig. 6. An excerpt of the attribute hierarchy and the yeast hierarchy in the $[M S]^{2} O$ domain component applied to the production of stabilized micro-organisms

notice that the hierarchical links between the Drying_resistance attribute and the attributes Acidifying_activity_losses, Cultivability_losses, Membrane_integrity_losses and Viability_losses in Figure 6 eases the querying of all observations associated with the Drying_resistance attribute.

\subsection{The Observation module}

According to definitions D2 and D3, observations are very important for the transformation process representation. They correspond to the experimental results. Each observation is performed by a material (i.e. a sensor) using a given method (see hasForM\&M property in Figure 4), on a step or on a mixture (see hasForAttribute property between FeatureOf Interest and ObservedAttribute, then hasForObservation property between ObservedAttribute and Observation in Figure 4) at a given scale (e.g. molecular, cellular, population) (see hasForScale property in Figure 4). It is important to stress on, as states in definition $\mathrm{D} 3$, that the multi scale property of $[M S]^{2} O$ is represented at the observation level. As a matter of fact, in our domain, what is important is to know at which scale a given experimental measure (i.e. an observation) is made, not to register the part-of links between different parts/scales of a product. Currently, the observations are made independently at different steps and different scales of the studied mixtures.

Example 4. The observations associated with the Cultivability_losses attribute are performed on mixtures at their population scale. In order to answer to $C Q_{3}$, we have to consider all the observations, associated with the Cultivabili$t y_{-}$losses attribute, which are performed on mixtures composed of a yeast during the drying step (see the excerpt of the yeast hierarchy in Figure 6).

An observation can be a raw observation or a computed observation. A computed observation is computed from raw observations (see isComputedFrom property in Figure 4). The observations are regrouped in a set of observations, which allows one to register that they were made together and represent the same set of experimental measures (see isMember0f property in Figure 4). 
An observation is characterized by the time when it is performed (see hasForTimeProperty property in Figure 4).

An observation and an input attribute are defined by their type (see hasForType property in Figure 4). This type can be either (i) a SimpleType, i.e. a quantity (e.g. temperature) or a symbolic concept (e.g texture), or (ii) a function composed of two operands (see Operand_1 and Operand_2 properties in Figure 4). The observation or the input attribute has then for value in the first case a single measure associated with a unit of measure, and, in the second case a multiple measure.

Example 5. The observations on the Membrane_integrity attribute, involved in $C Q_{2}$, are computed from the raw observations associated with the Fluorescence_intensity attribute. Each raw observation on the Fluorescence_intensity attribute has for type a function between the quantity Fluorescence_intensity_quantity and the quantity NumberOfCells. Each computed observation on the Membrane_integrity attribute has a simple type, the boolean symbolic concept. It has for value a boolean value, with the unit of measure 'no_unit'.

\section{The ontology implementation and validation}

The current version of $[M S]^{2} O$ core component implemented in OWL is available at http://lovinra.inra.fr/2015/12/16/multi-scale-multi-step-ontology/. Time ontology $\mathrm{y}^{8}$ recommended by the $\mathrm{W} \mathrm{C}^{9}$ is used to represent the temporal concepts. For the measure part we reuse the modeling of the measures with their units from the @Web platform ${ }^{10}[4]$ which was inspired from $\mathrm{OM}^{11}$.

Available data concerning two production processes with two itineraries each were structured using the $[M S]^{2} O$ vocabulary and are stored into 162 EXCEL files. For each production process,

- 1 file describes the process,

- 66 files contain experimental observations, where the biggest experimental observation contains 297 results' raws,

- 4 files contain the mixture composition,

- 9 files describe the steps, and

- 1 file contains the environmental impact information.

Example 6. Figure 7 gives a screen-shot of an excerpt of the EXCEL file which describes a process.

These EXCEL files allow us to build the $[M S]^{2} O$ domain component and to validate that all available data in the use case can be represented as instances of $[M S]^{2} O$. To validate our conceptualization, we have checked that the SPARQL

\footnotetext{
${ }^{8}$ http://www.w3.org/TR/owl-time/

${ }^{9}$ World Wide Web Consortium. http://www.w3.org/

${ }^{10}$ http://www6.inra.fr/cati-icat-atweb

11 http://www.wurvoc.org/vocabularies/om-1.8/
} 


\begin{tabular}{|c|c|c|c|c|c|c|c|}
\hline Date & $2015-06-28$ & & & & & & \\
\hline $\begin{array}{c}\text { Project } \\
\text { name }\end{array}$ & LIONES & & & & & & \\
\hline $\begin{array}{c}\text { Microorgani } \\
\text { sm (species, } \\
\text { stem) }\end{array}$ & $\begin{array}{c}\text { Saccharomyces } \\
\text { cerevisiae - CBS } \\
8066\end{array}$ & & & & & & \\
\hline \begin{tabular}{|c|} 
Code \\
échantillon \\
\end{tabular} & $\begin{array}{l}\text { 2015-06-28- } \\
\text { LIONES-001 }\end{array}$ & & & & & & \\
\hline Step & SubStep & \begin{tabular}{|c|} 
time \\
harvest
\end{tabular} & Measure & Measure level & Scale & \begin{tabular}{|c|} 
File \\
number
\end{tabular} & Type of data \\
\hline Fermentation & \begin{tabular}{|c|}
$\begin{array}{c}\text { Preparation of the } \\
\text { culture mixture }\end{array}$ \\
\end{tabular} & NA & Composition & Mixture & & 1 & raw \\
\hline Fermentation & \begin{tabular}{|c|} 
Sterilisation of the \\
culture mixture
\end{tabular} & NA & Conduite & Step & & 1 & raw \\
\hline Fermentation & Culture & $24 \mathrm{~h}$ & Conduite & Step & & 3 & raw \\
\hline \begin{tabular}{|l|} 
Fermentation \\
\end{tabular} & Culture & $24 \mathrm{~h}$ & Cultivability & Mixture & Population & 1 & raw-computed \\
\hline \begin{tabular}{|l|} 
Fermentation \\
\end{tabular} & Cleaning & NA & Composition & Mixture & Mixture & 1 & raw \\
\hline Concentration & NA & $20 \mathrm{~min}$ & Conduite & Step & & 1 & raw \\
\hline $\begin{array}{l}\text { Drying into } \\
\text { stove }\end{array}$ & NA & $75 \mathrm{~min}$ & Conduite & Step & & 2 & raw \\
\hline $\begin{array}{l}\text { Drying into } \\
\text { stove }\end{array}$ & NA & $75 \mathrm{~min}$ & GSH intracellular & Mixture & Cellular & 1 & raw-computed \\
\hline \begin{tabular}{c|} 
Drying into \\
fluidized bed
\end{tabular} & $60^{\circ} 60^{\prime}$ & $60 \mathrm{~min}$ & Conduite & Step & & 1 & raw \\
\hline $\begin{array}{c}\text { Drying into } \\
\text { fluidized bed }\end{array}$ & $60^{\circ} 60^{\prime}$ & $60 \mathrm{~min}$ & GSH intracellular & Step & Cellular & 1 & raw-computed \\
\hline Storage & NA & NA & $\begin{array}{c}\text { Energy } \\
\text { consumption }\end{array}$ & Step & & 1 & computed \\
\hline
\end{tabular}

Fig. 7. An excerpt of the EXCEL file which describes a process

queries deduced from the competency questions presented in Section 2.2 have answers in a subset of OWL instances built from the EXCEL files. A knowledge base built from EXCEL files is currently in progress.

Example 7. The SPARQL queries to answer to $C Q_{3}$ and $C Q_{5}$ presented in examples 3 and 4 are:

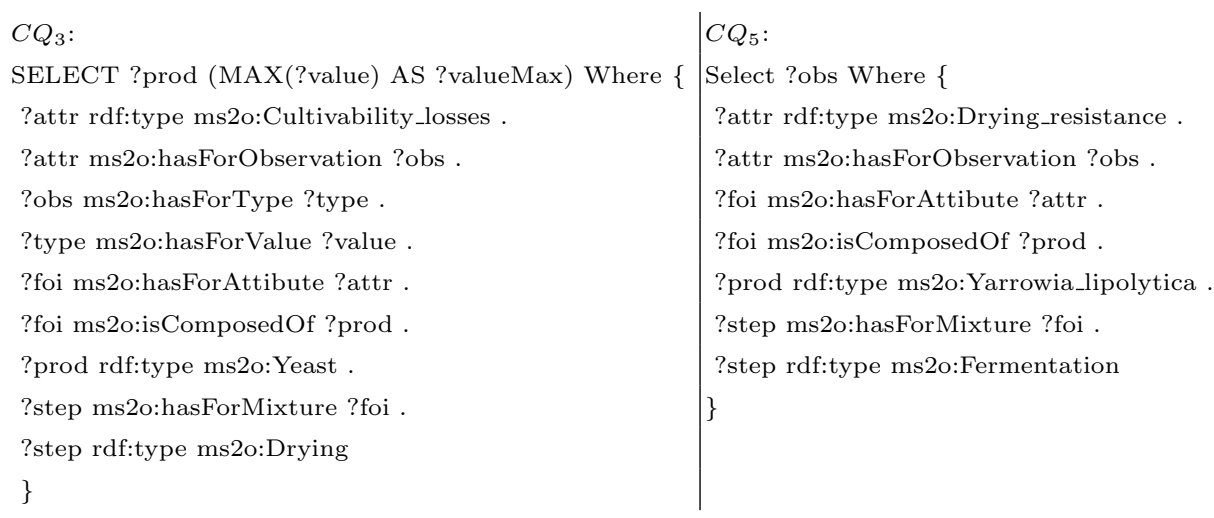

\section{Conclusions and Further Work}

In this paper, we presented the building of $[M S]^{2} O$, a Multi-Scale and Multi-Step Ontology for transformation processes, applied to the production of stabilized 
micro-organisms use case, performed at INRA (French National Institute for Agricultural Research). To build $[M S]^{2} O$, we have followed the NeOn methodology. We detailed the $[M S]^{2} O$ core component in which a transformation process is composed of steps, non necessarily in sequence, that allows inputs to be transformed in several different outputs. We stated that a transformation process relies on experimental observations that are performed either on a mixture (i.e. a set of combined products) at a certain scale during a certain step or on a step, the mixture being transformed during the steps of the process.

Future work is to define the module Material and Method and its connections with the other modules. We are currently investigating if the Semantic Sensor Network ontology ${ }^{12}$ recommended by the W3C could be used. We are also evaluating how to enrich the product hierarchy with the FAO thesauri, AGROVOC ${ }^{13}$ and the EFSA classification, FoodEx $2^{14}$.

In order to test the genericity of our $[M S]^{2} O$ core component modeling we are currently investigating how to use it in an other project concerning transformation processes on dairy gels.

$[M S]^{2} O$ ontology aims to play a key role as the representation layer of the querying and simulation systems of the LIONES project. This leads to the possibility of comparing different production scenarios and suggesting improvements while increasing efficiency (e.g. costs for the company). Moreover modeling the production processes of multi-scale objects may help domain experts to discover new semantic links between concepts and perform a cause effect analysis.

Acknowledgments We are very grateful for the valuable inputs from all the domain experts partners involved in CellExtraDry French national project.

\section{References}

1. Catherine Béal, Michèle Marin, Éloi Fontaine, Fernanda Fonseca, and JeanPhilippe Obert. Production et conservation des ferments lactiques et probiotiques. In C. Georges and L. François-Marie, editors, Bactéries lactiques. De la génétique aux ferments, pages 661-786. Lavoisier, 2008.

2. Christian Bizer. Interlinking scientific data on a global scale. Data Science Journal, 12:GRDI6-GRDI12, 2013.

3. Eva Blomqvist, Pascal Hitzler, Krzysztof Janowicz, Adila Krisnadhi, Tom Narock, and Monika Solanki. Considerations regarding ontology design patterns. Semantic Web, 7(1):1-7, 2016.

4. Patrice Buche, Juliette Dibie-Barthélemy, Liliana Ibanescu, and Lydie Soler. Fuzzy web data tables integration guided by an ontological and terminological resource. IEEE Trans. Knowl. Data Eng., 25(4):805-819, 2013.

5. María del Carmen Suárez-Figueroa, Asunción Gómez-Pérez, and Mariano Fernández-López. The NeOn methodology for ontology engineering. In María

\footnotetext{
12 http://www.w3.org/2005/Incubator/ssn/XGR-ssn-20110628/

${ }^{13}$ http://aims.fao.org/vest-registry/vocabularies/agrovoc-multilingual-agriculturalthesaurus

14 http://www.efsa.europa.eu/fr/datex/datexfoodclass
} 
del Carmen Suárez-Figueroa, Asunción Gómez-Pérez, Enrico Motta, and Aldo Gangemi, editors, Ontology Engineering in a Networked World., pages 9-34. Springer, 2012.

6. Julie Gautier, Stéphanie Passot, Caroline Pénicaud, Hervé Guillemin, Stéphanie Cenard, Pascale Lieben, and Fernanda Fonseca. A low membrane lipid phase transition temperature is associated with a high cryotolerance of Lactobacillus delbrueckii subspecies bulgaricus CFL1. Journal of Dairy Science, 96(9):5591-5602, 2013.

7. Tonci Grubic and Ip-Shing Fan. Supply chain ontology: Review, analysis and synthesis. Computers in Industry, 61(8):776 - 786, 2010. Semantic Web Computing in Industry.

8. Nicola Guarino, Daniel Oberle, and Steffen Staab. What is an ontology? In Steffen Staab and Rudi Studer, editors, Handbook on Ontologies, International Handbooks on Information Systems, pages 1-17. Springer Berlin Heidelberg, 2009.

9. Krzysztof Janowicz, Adila Krisnadhi, Yingjie Hu, Sangwon Suh, Bo Pedersen Weidema, Beatriz Rivela, Johan Tivander, David E. Meyer, Gary Berg-Cross, Pascal Hitzler, Wesley Ingwersen, Brandon Kuczenski, Charles Vardeman, Yiting Ju, and Michelle Cheatham. A minimal ontology pattern for life cycle assessment data. In Eva Blomqvist, Pascal Hitzler, Adila Krisnadhi, Tom Narock, and Monika Solanki, editors, Proceedings of the 6th Workshop on Ontology and Semantic Web Patterns (WOP 2015) co-located with the 14th International Semantic Web Conference (ISWC 2015), Bethlehem, Pensylvania, USA, October 11, 2015., volume 1461 of CEUR Workshop Proceedings. CEUR-WS.org, 2015.

10. Aunur-Rofiq Muljarto, Jean-Michel Salmon, Pascal Neveu, Brigitte Charnomordic, and Patrice Buche. Ontology-based model for food transformation processes - application to winemaking. In Sissi Closs, Rudi Studer, Emmanouel Garoufallou, and Miguel-Ángel Sicilia, editors, Metadata and Semantics Research - 8th Research Conference, MTSR 2014, Karlsruhe, Germany, November 27-29, 2014. Proceedings, volume 478 of Communications in Computer and Information Science, pages 329-343. Springer, 2014.

11. Caroline Pénicaud, Sophie Landaud, Frédéric Jamme, Pauline Talbot, Marielle Bouix, Sarrah Ghorbal, and Fernanda Fonseca. Physiological and biochemical responses of Yarrowia lipolytica to dehydration induced by air-drying and freezing. PLoS ONE, 9:e111138, 2014.

12. Marco Rospocher, Chiara Ghidini, and Luciano Serafini. An ontology for the business process modelling notation. In Formal Ontology in Information Systems Proceedings of the Eighth International Conference, FOIS 2014, September, 22-25, 2014, Rio de Janeiro, Brazil, pages 133-146, 2014.

13. Charles Vardeman, Adila Krisnadhi, Michelle Cheatham, Krzysztof Janowicz, Holly Ferguson, Pascal Hitzler, Aimee Buccellato, Krishnaprasad Thirunarayan, Gary Berg-Cross, and Torsten Hahmann. An ontology design pattern for material transformation. In Proceedings of the 5th Workshop on Ontology and Semantic Web Patterns (WOP2014) co-located with the 13th International Semantic Web Conference (ISWC 2014), Riva del Garda, Italy, October 19, 2014., pages 73-77, 2014.

14. Hélène Velly, Marielle Bouix, Stéphanie Passot, Caroline Pénicaud, Hélène Beinsteiner, Sarrah Ghorbal, Pascale Lieben, and Fernanda Fonseca. Cyclopropanation of unsaturated fatty acids and membrane rigidification improve the freeze-drying resistance of Lactococcus lactis subsp. lactis TOMSC161. Applied Microbiology and Biotechnology, 99(2):907-918, 2015. 